\title{
Educating healthcare providers in the delivery of genomic medicine
}

\author{
Jeanette McCarthy*,1 \& George P Patrinos ${ }^{2,3,4}$ \\ ${ }^{1}$ Center for Applied Genomics \& Precision Medicine, Duke University, Durham, NC 27516, USA \\ ${ }^{2}$ Department of Pharmacy, School of Health Sciences, University of Patras, Patras, Greece \\ ${ }^{3}$ Zayed Center of Health Sciences \& Department of Pathology, United Arab Emirates University, Al-Ain, UAE \\ ${ }^{4}$ Department of Pathology, College of Medicine \& Health Sciences, United Arab Emirates University, Al-Ain, UAE \\ *Author for correspondence: jeanette.mccarthy@duke.edu
}

\begin{abstract}
"The contributors to this special focus issue of Personalized Medicine are leading the way to improving genomic literacy and ultimately to the advancement of genomic medicine."
\end{abstract}

First draft submitted: 1 March 2019; Accepted for publication: 4 March 2019; Published online: 13 June 2019

Keywords: education $\bullet$ genomic literacy $\bullet$ personalized medicine $\bullet$ precision medicine

In the past several decades, our understanding of the genetic basis of diseases has grown exponentially. Coupled with advances in sequencing technology, this knowledge has led to the development of molecular genetic tests for diagnosing disease and identifying individuals at risk of genetic disorders [1]. Despite our ability to solve diagnostic dilemmas in children, screen for genetic disorders in utero and identify individuals at risk of hereditary cancers, cardiovascular and other diseases, uptake and acceptance of genetic testing in clinical practice remain low.

Some have attributed the lack of uptake to undereducated healthcare providers and have called for improvements in healthcare professional training in genomics. But according to healthcare professionals' opinion, genomic medicine is often not on the top of their list of concerns. There may be more pressing, modifiable risk factors to address in their patients like obesity, poverty, opioid use, not to mention chronic diseases and cancer. Some perceive genetic testing as too expensive, invalid or outside of their domain of expertise. Some providers might be unaware of how far the field has come [2]. Others, uncomfortable with their own lack of genomic literacy and skills implementing genetic testing, may prefer to defer to a genetic counselor [3].

What healthcare providers need to understand is that genomic medicine is being practiced today and will continue to permeate medicine and touch every healthcare provider at one time or another, regardless of specialty. This may not be evident if healthcare providers and/or other stakeholders are outside of a major academic medical center, but programs like the one at Mission Health System, described by Dressler $e t a l$. in this issue, serve as a model for diffusion of the latest genomic medicine innovations into the community setting [4]. Understanding genomics and genetic testing will be an essential skill for 21 st century medicine. Education that addresses improved genomic literacy, development of practical skills for implementing genomic testing in the clinic and increased awareness of how genomics can be used to improve health, will best prepare the healthcare workforce for a future that includes genomic medicine $[5,6]$.

Exactly how we educate medical professionals in the rapidly evolving field of genomic medicine is the subject of this special issue of Personalized Medicine. Authors from leading healthcare institutions share their innovative strategies for teaching providers during undergraduate medical education and beyond. These programs incorporate different strategies to engage learners in a clinically meaningful way and utilize different online platforms to accommodate busy working professionals.

Some of the strategies involve personal genetic testing - offering genetic testing to the learners themselves. Ta $e t$ al. describe, among other things, how incorporating pharmacogenomic testing of PharmD students at University of California, San Francisco (UCSF), USA improved attitudes and confidence about pharmacogenomic testing [7]. However, Hyland et al. surveyed undergraduate medical student education programs in genomics and genetics, and 
found that cost and ethical concerns have prevented widespread use of personal genetic testing in many healthcare professional classrooms [8].

Hyland et al. describe other nonlecture-based active learning methods that are being used to boost student understanding, confidence in and engagement with this material, including flipped classroom and case-based learning [8]. Case-based learning is also at the heart of the educational several free CME-eligible modules developed by the Jackson Laboratory (JAX), American Medical Association (AMA) and Scripps Research Translational Institute (SRTI), which cover various applications of genomics in medicine. Edelman et al. describe the effectiveness of the two JAX/AMA/SRTI online, interactive cased-based modules for somatic tumor testing [9].

Online education really took off in the mid 2010s when platforms like Coursera began offering free online courses taught by experts at reputable academic institutions around the world, thereby democratizing education. The scope and availability of online learning opportunities in genomic medicine, pharmacogenomics and bioinformatics are the theme of the article by Tsermpini et al. [10]. They identified 18 individual courses and 24 programs leading to either a masters or postgraduate certificate, a relatively small number considering the number of healthcare providers who need education. Bioinformatics education is the topic of the paper by Moore et al., who describe the need for training of next-generation clinical scientists to transform biomedical big data into new tests and treatment.

The need for education in genomic medicine is clear and much progress is being made identifying the best platforms and teaching methods to reach healthcare providers at various stages of their career. The contributors to this special focus issue of Personalized Medicine are leading the way to improving genomic literacy and ultimately to the advancement of genomic medicine.

Financial \& competing interests disclosure

A grant from the Ubiquitous Pharmacogenomics (U-PGX) project (European Commission 668353) was received for the creation of this manuscript. The authors have no other relevant affiliations or financial involvement with any organization or entity with a financial interest in or financial conflict with the subject matter or materials discussed in the manuscript apart from those disclosed.

No writing assistance was utilized in the production of this manuscript.

\section{References}

1. Manolio TA, Abramowicz M, Al-Mulla F et al. Global implementation of genomic medicine: we are not alone. Sci. Transl. Med. 7 , 290ps13 (2015).

2. Kampourakis K, Vayena E, Mitropoulou C et al. Key challenges for next generation pharmacogenomics. EMBO Rep. 15, 472-476 (2014).

3. Mai Y, Mitropoulou C, Papadopoulou XE et al. Critical appraisal of the views of healthcare professionals with respect to pharmacogenomics and personalized medicine in Greece. Per. Med. 11, 15-26 (2014).

4. Dressler L, Bell GC, Schuetze DP et al. Implementing a personalized medicine cancer program in a community cancer system. Per. Med. 16(4), (2019).

5. Reydon TA, Kampourakis K, Patrinos GP. Genetics, genomics and society: the responsibilities of scientists for science communication and education. Per. Med. 9, 633-643 (2012).

6. Kampourakis K, Reydon TA, Patrinos GP, Strasser BJ. Genetics and society - educating scientifically literate citizens. Sci. Edu. 23, 251-258 (2014).

7. Ta R, Cayabyab MAS, Coloso R. Precision medicine: a call for increased pharmacogenomic education. Per. Med. 16(4), 233-245 (2019).

8. Hyland C, Garber K, Dasgupta S. From helices to health: undergraduate medical education in genetics and genomics. Per. Med. 16(4), 211-220 (2019).

9. Edelman E. Provider engagement in precision oncology education: an exploratory analysis of online continuing medical education data. Per. Med. 16(4), 199-209 (2019).

10. Tsermpini EE, Stamopoulou T, Kordou Z, Barba E, Stathoulias A, Patrinos GP. Continuous pharmacogenomics and genomic medicine education for healthcare professionals through electronic educational courses. Per. Med. 16(4), 189-193 (2019). 